\title{
Ten-year results of concentrated autologous bone marrow aspirate transplantation for osteonecrosis of the femoral head: a retrospective study
}

Yohei Tomaru', Tomokazu Yoshioka² ${ }^{2}$ D, Hisashi Sugaya ${ }^{2}$, Hiroshi Kumagai ${ }^{1}$, Kojiro Hyodo $^{1}$, Katsuya Aoto ${ }^{1}$, Hiroshi Wada', Hiroshi Akaogi ${ }^{1}$, Masashi Yamazaki ${ }^{1}$ and Hajime Mishima ${ }^{1}$

\begin{abstract}
Background: Idiopathic osteonecrosis of the femoral head (ONFH) occurs at a relatively younger age. It is therefore important to prevent the resultant femoral head collapse and requirement of total hip arthroplasty in these patients. In 2003, we initiated concentrated autologous bone marrow aspirate transplantation (CABMAT), a joint-preserving treatment for ONFH, at our institution. Here, we report the long-term results of CABMAT treatment.

Methods: We retrospectively collated and analyzed the demographic and treatment data of 69 patients (109 hips) with idiopathic ONFH treated with CABMAT between April 2003 and April 2008.

Results: Totally, 44 patients (21 men, 23 women, 80 hips) completed the 10-year follow-up. The follow-up rate was $73.4 \%$, and the mean follow-up period was 12.0 (range, 10.0-15.4) years. The mean age of the patients was 42.2 (range, 16.3-70.5) years. Using the Association Research Circulation Osseous (ARCO) classification system for preoperative analysis, 12,31,32, and 5 hips were classified as stages 1, 2, 3, and 4, respectively. The overall rate of conversion to total hip arthroplasty (THA) was 34\% (27/80 hips). In a multivariate regression analysis, the preoperative stage of ONFH and the body mass index were found to correlate significantly with conversion to THA. Totally, 43 hips (of 80) were classified as belonging to the pre-collapse stage (i.e., stages 1 or 2). The overall collapse rate and the THA-conversion rate of these hips were estimated to be $49 \%$ (21/43) and 14\% (6/43), respectively.

Conclusions: On the basis of our long-term findings, the minimally invasive and feasible CABMAT therapy can be utilized as one of a joint-preserving treatment for ONFH.
\end{abstract}

Keywords: Bone marrow aspirate concentrate, Hip preserving surgery, Osteonecrosis of the femoral head

\section{Background}

Idiopathic osteonecrosis of the femoral head (ONFH) is a refractory hip disorder, which leads to femoral head collapse and hip osteoarthritis. ONFH is defined as an aseptic, ischemic, and atraumatic necrosis of the femoral head and is mainly associated with corticosteroid use and alcohol consumption in Japan [1]. The annual incidence of ONFH in Japan is estimated to be 2.58 cases

\footnotetext{
* Correspondence: yoshioka@md.tsukuba.ac.jp

2Division of Regenerative Medicine for Musculoskeletal System, Department of Orthopaedic Surgery, Faculty of Medicine, University of Tsukuba, 1-1-1 Tennodai, Tsukuba, Ibaraki 305-8575, Japan

Full list of author information is available at the end of the article
}

per 100,000 (range, 1.54-3.66) person-years [2]. The average age at presentation is 50.4 years; ONFH thus involves relatively young and active patients [3]. Total hip arthroplasty (THA) is widely performed in patients with femoral head collapse. However, considering the possibilities of both implant dislocation and expiration, it is desirable to avoid THA at a young age. In particular, compared to osteoarthritis, osteonecrosis is reportedly a high-risk disease, adversely affecting the long-term durability of THA [4]. To avert the requirement for THA at a young age, it is important to prevent femoral head collapse or to minimize its extent. Prevention of both femoral collapse and conversion to THA are the main

(c) The Author(s). 2019 Open Access This article is distributed under the terms of the Creative Commons Attribution 4.0 International License (http://creativecommons.org/licenses/by/4.0/) which permits unrestricted use, distribution, and reproduction in any medium, provided you give appropriate credit to the original author(s) and the source, provide a link to the Creative Commons license, and indicate if changes were made. The Creative Commons Public Domain Dedication waiver (http://creativecommons.org/publicdomain/zero/1.0/) applies to the data made available in this article, unless otherwise stated. 
purposes of joint-preserving treatments, which include femoral and innominate osteotomy, mesenchymal stem cell (MSC) transplantation, and growth factor administration. Several previous studies have also reported the efficacy of bone marrow transplantation in ONFH [5-7].

We initiated concentrated autologous bone marrow aspirate transplantation (CABMAT) for the treatment of $\mathrm{ONFH}$ in 2003 [8]. As a part of the transplant procedure, bone marrow is aspirated from the iliac crest of the affected patient and centrifuged twice to separate the buffy coat. The femoral necrotic area is drilled into, and the extracted buffy coat transplanted at the site [8]. The buffy coat extract contains bone marrow cells, MSCs, osteoprogenitor cells, and growth factors [9]. In 2017, we reported the results of CABMAT after a mean follow-up period of 5 years in patients with asymptomatic ONFH [10, 11]. This study aimed to report the results of management of ONFH with CABMAT for $>10$ years.

\section{Methods}

In this retrospective study, we followed-up patients treated with CABMAT for ONFH over a minimum 10-year period to evaluate its long-term outcomes. The study was conducted at the University of Tsukuba, Japan, under the supervision of Professor Dr. Masashi Yamazaki and Associate professor Dr. Hajime Mishima.

\section{Patients}

We retrospectively analyzed data of 69 patients (109 hips) diagnosed with idiopathic ONFH, treated using CABMAT, between April 2003 and April 2008. The basic indication for CABMAT was ONFH at the pre-collapse or collapse stage (with the affected portion $<3 \mathrm{~mm}$ in diameter), i.e., stage 1 to stage $3 \mathrm{~A}$ of the Association Research Circulation Osseous (ARCO) classification [12]. Although there were no clinical and radiological data on CABMAT for stages $3 \mathrm{~B}, 3 \mathrm{C}$, and 4 available in the initial period of the study, CABMAT was also performed for patients affected by these stages of ONFH. No patients were treated using the core decompression procedure only.

\section{ONFH diagnosis and classification}

We used the ARCO classification system for the diagnosis, analysis, and classification of ONFH [12]. According to this classification, ONFH is classified into stages 1,2 , 3 , and 4 according to the X-ray, computed tomography, scintiscan, and magnetic resonance imaging (MRI) findings. In stage 0 , no abnormal findings are seen on any of the imaging studies. In stage 1, abnormal findings are seen only on scintiscan and/or MRI. In stage 2, radiographic abnormalities (e.g. sclerosis, osteolysis, and focal porosis) without crescent sign and collapse are seen. In stage 3, radiographic abnormalities (crescent sign and/or collapse) without progression to osteoarthritis are observed. In stage 4, progression to osteoarthritis is seen.

\section{Bone marrow aspiration, concentration, and transplantation technique}

Several methods of bone marrow transplantation for ONFH exist. Generally, the approaches can be classified as concentration and culture methods. The culture method can deliver a larger number of MSCs compared to the concentration method. However, safety, quality of MSCs, and cost are significant issues [13]. Furthermore, the concentration method can deliver not only MSCs, but also cytokines and growth factors. For these reasons, the concentration method was adopted at our institution and the novel CABMAT approach was developed. CABMAT was performed according to our established protocol, as reported in an earlier study [8]. CABMAT is a single-step, minimally invasive surgical procedure as compared with either osteotomy or THA and does not require a special device, such as a cell sorter.

The marrow was aspirated from both iliac crests of each patient, using a bone marrow harvesting needle (Medical Device Technologies, Inc., Gainesville, FL, USA) and transferred into a bone marrow collection bag. After centrifugation at $1200 \mathrm{~g}$ for $10 \mathrm{~min}$ (Centrifuge Kubota 9800, Kubota, Japan), erythrocytes were transferred from the main bag to a satellite bag. After a second round of centrifugation ( $3870 \mathrm{~g}$ for $7 \mathrm{~min}$ ), the plasma was transferred from the main bag to another satellite bag, which left the buffy coat layer in the main bag.

During transplantation, we first performed core decompression of the femoral head using a trephine with a thickness of $4.8 \mathrm{~mm}$ (Iso Medical Systems, Tokyo, Japan). The drill was inserted percutaneously into the center of the necrotic area. After core decompression, drilling was performed multiple times using a 2.4- $\mathrm{mm}$ diameter Kirschner wire. To perforate the interface between the normal bone and necrotic bone, the surgeon drilled repeatedly at sites anteromedial and posterolateral to the core decompression tract. We then used a monitor with biplane fluoroscopic guidance to transplant the buffy coat at the drilled sites.

\section{Colony-forming-unit-fibroblast (CFU-F) assay}

We performed the CFU-F assay to evaluate the presence of MSCs in the buffy coat. The analysis was also performed according to our previously published method [8]. After 2 weeks of culture at $37^{\circ} \mathrm{C}$ in a humidified atmosphere of $5 \%$ carbon dioxide, the number of colonies $>2 \mathrm{~mm}$ in diameter were counted. The number of MSCs was expressed as the number of CFU-Fs per $10^{6}$ nucleated cells. Since the CFU-F assay had not been performed during the initial period of the study, we could only collect these data for 53 of 80 hips (66\%). As this 
was a significant loss, CFU-F assay data were not included in the statistical analyses.

\section{Outcome measures}

The rate of conversion of the treatment protocol from CABMAT to THA was set as the primary endpoint of this study. The indication for THA in this study was the development of stage 4, painful ONFH. The percentage of hips developing femoral head collapse and factors predictive of conversion to THA were set as secondary endpoints. Treatment efficacy was estimated using the rate of conversion to THA, which was indicative of the effect of CABMAT on the natural history of ONFH. All hips were accordingly classified into THA-conversion or THA non-conversion groups for the univariable analysis. Univariable and multivariable analyses were also performed to evaluate factors predictive of THA conversion. The mean age, body mass index (BMI), and follow-up period were compared between both groups using the unpaired $t$ test. The chi-square test was used to compare the sex distribution of the patients between both groups. We compared etiologies and stages of ONFH (as per the ARCO classification) between the two groups using residual analysis. THA was set as the objective variable for the multivariable logistic analysis. Factors including age, sex, etiology, BMI, follow-up period, and preoperative stage were set as the explanatory variables. A $p$-value < 0.05 was considered statistically significant. All statistical analyses were performed with SPSS Statistics version 15 (IBM, Armonk, NY) software.

\section{Radiographic evaluation of femoral head collapse}

Anteroposterior and lateral radiography of the treated hip was performed at regular intervals during the patients' follow-up hospital visits. The presence of femoral head collapse was determined using circular template overlays [14] on anteroposterior or lateral radiographs, irrespective of the site of the collapse. All radiographs were independently evaluated by two specialist orthopedic surgeons.

\section{Results}

We were able to conduct follow-up evaluations in 44 of the 69 patients ( 21 men, 23 women; 80 of 109 hips) for $>10$ years. The follow-up rate was $73.4 \%(80 / 109)$. The mean age of the patients was 42.2 (range, 16.3-70.5) years, and the mean follow-up period was 12.0 (10.015.4) years. The mean CFU-F count per $10^{6}$ nucleated cells was $2.33 \pm 2.67$. The overall THA-conversion rate was $34 \%(27 / 80)$. The mean period before conversion to THA was 4.4 (range, 0.5-15; median, 2.9) years. The survival curves are shown in Fig. 1 . The rate of conversion to THA was $25 \%(3 / 12), 10 \%(3 / 31), 50 \%(16 / 32)$, and $100 \%(5 / 5)$ for ONFH stages $1,2,3$, and 4 , respectively. THA conversion rate was $14 \%(6 / 43)$ for pre-collapsed ONFH stages (i.e., stages 1 or 2). Characteristics of the THA-conversion and non-conversion groups are shown in Table 1. Factors including BMI and rate of occurrence of ONFH stages $3 \mathrm{~B}$ and 4 were significantly higher in the THA-conversion group. The preoperative stage of osteonecrosis and the patient's BMI were found to be significantly correlated with conversion to THA in the multivariable statistical analysis. Metabolic syndrome (diabetes mellitus, hypertension, and hyperlipidemia) was present in 40\% (21/ 53) and $44 \%(12 / 27)$ of the THA non-converted and THA converted groups, respectively, with no significant difference between them (chi-square test, $p=0.67$ ).

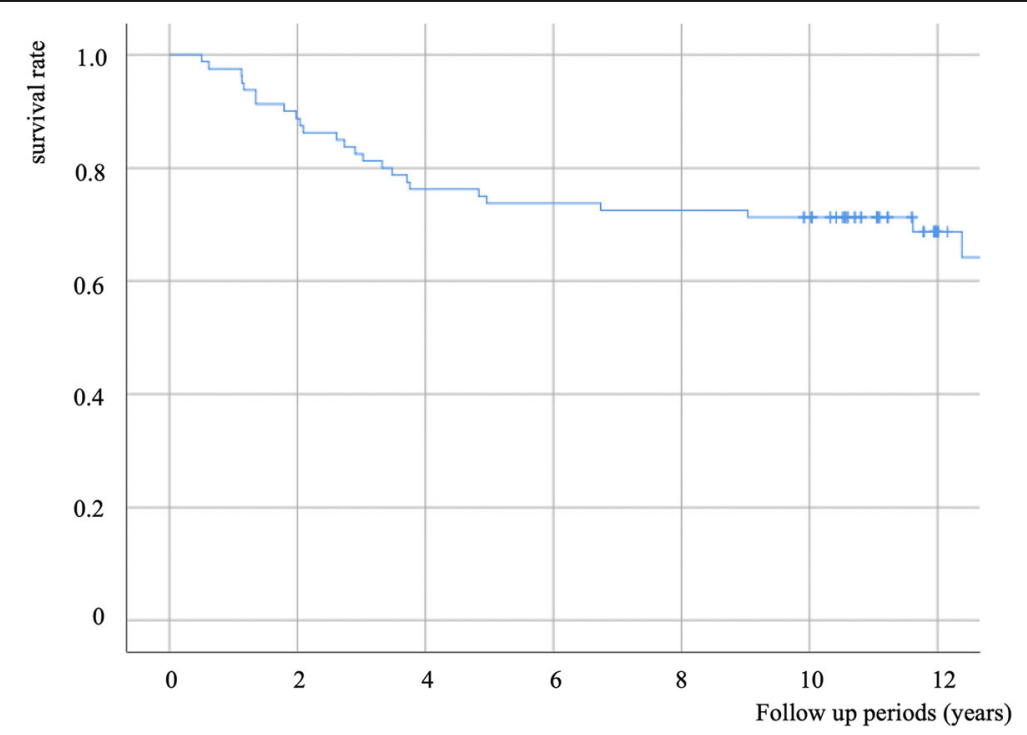

Fig. 1 Survival curve (end point: conversion to total hip arthroplasty) 
Table 1 Comparison of characteristics of the THA-conversion and THA non-conversion groups

\begin{tabular}{|c|c|c|c|c|}
\hline & $\begin{array}{l}\text { THA }{ }^{\mathrm{b}} \text {-conversion } \\
\text { group }\end{array}$ & $\begin{array}{l}\text { THA }{ }^{\mathrm{b}} \\
\text { non-conversion group }\end{array}$ & $P$-value & Statistical method \\
\hline$n$ & 27 & 53 & - & - \\
\hline Men/Women & $11 / 16$ & $30 / 23$ & n.s. & $x^{2}$ test \\
\hline Age & $43.7(21.5-70.5)$ & $41(16-71)$ & n.s. & $t$-test \\
\hline BMl & $24(20-32)^{a}$ & $21.9(17-30)$ & $p<0.01$ & t-test \\
\hline Presence of metabolic syndrome & $40 \%(21 / 53)$ & $44 \%(12 / 27)$ & n.s. & $x^{2}$ test \\
\hline Follow up period & $13(11-15)$ & $11.8(10.0-15.0)$ & n.s. & t-test \\
\hline \multicolumn{5}{|l|}{ Etiology } \\
\hline Steroid & 19 (70\%) & $37(70 \%)$ & n.s. & Residual analysis \\
\hline Alcohol & $5(19 \%)$ & $14(26 \%)$ & & \\
\hline Idiopathic & $3(11 \%)$ & $2(4 \%)$ & & \\
\hline \multicolumn{5}{|l|}{ Preoperative stage } \\
\hline 1 & 3 & 9 & n.s. & \\
\hline 2 & 3 & $28^{\mathrm{a}}$ & $p<0.01$ & \\
\hline 3 & 16 & 16 & n.s. & \\
\hline 4 & $5^{a}$ & 0 & $p<0.01$ & \\
\hline
\end{tabular}

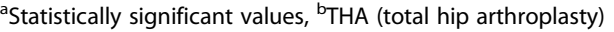

Of the 80 hips, 43 were classified as belonging to the pre-collapse stage (i.e., stages 1 or 2). The overall collapse rate for these hips was 49\% (21/43). The collapse rates of hips belonging to ONFH stages 1 and 2 were $50 \%(6 / 12)$ and $48 \%(15 / 31)$, respectively. THA conversion rate in pre-collapse stage (i.e., stages 1 or 2) was $14 \%(6 / 43)$, respectively. There were no cases of tumorigenic transformation or infection following CABMAT.

\section{Discussion}

We studied the long-term outcomes of CABMAT. In summary, the overall conversion to THA rate was $34 \%$ (27/80 hips). Preoperative stage and BMI were found to correlate significantly with conversion to THA in the multivariate regression analysis. Forty-three hips (of 80) were classified as belonging to the pre-collapse stage (i.e., stages 1 or 2). The overall collapse and THA-conversion rates of these hips were $49 \%(21 / 43)$ and $14 \%(6 / 43)$, respectively.

The exact pathophysiology of ONFH is still unclear. Studies have suggested genetic predisposition, metabolic factors, mechanical stress, vascular failure, and raised intraosseous pressure as possible causes [15]. Intake of corticosteroids and excessive alcohol not only induce development of the fat-storing phenotype among the bonemarrow progenitor cells, which leads to an insufficient supply of normal cells [6], but also cause fat embolisms and arteriosclerosis, which result in a reduced blood supply to necrotic areas [16]. Corticosteroids reportedly have an adverse effect on bone renewal by decreasing the number of progenitor cells [17]. Kuroda et al. reported good clinical outcomes of local administration of gelatin hydrogel impregnated with recombinant human fibroblast growth factor (FGF)-2 for the treatment of the pre-collapse stage of ONFH [18]. This suggests that FGF pathology plays a role in necrosis, and its replacement with functional growth factor will prevent femoral head collapse. Considering that osteonecrosis may be caused by vascular failure, FGF failure, raised intraosseous pressure, and an insufficiency of progenitor cells at the necrotic sites, we used CABMAT as a therapeutic modality. In CABMAT, the isolated buffy coat, which contains MSCs and various growth factors (e.g., platelet-derived growth factors, FGFs, and transforming growth factor$\beta)$, is transplanted to the necrotic area after core decompression [9]. Core decompression effectively lowers the intraosseous pressure within the necrotic area, thereby inducing migration of MSCs and various growth factors from healthy bone into the necrotic tissue [19].

The collapse and THA-conversion rates in the pre-collapse stages were found to be $49 \%(21 / 43)$ and $14 \%$ (6/ $43)$, respectively. The overall THA-conversion rate was $34 \%(27 / 80)$. There are several reports on the natural history and progression of ONFH. The rate of conversion to THA in our present study was lower than that reported previously [20], while no significant difference was observed as compared to that reported by others $[21,22]$ (Table 2).

Hernigou et al. reported a THA-conversion rate of $18 \%$ in $\sim 13$ years [6], which was lower than the rate observed in this study. By definition, the protocol of Hernigou et al. only included radiographic stages 1 and 2 [6]. In the present study, the THA-conversion rate in the pre-collapse stages ( 1 and 2$)$ was $14 \%(6 / 43)$, which was 
Table 2 Comparison of our study results with the natural history of ONFH and outcomes of other treatments

\begin{tabular}{|c|c|c|c|c|c|}
\hline & Treatment & Number of hips & Follow-up period (years) & Collapse Rate (\%) & THA -conversion rate (\%) \\
\hline this study & $C D^{a}+B M C^{b}$ & 80 & 12 & $60 \%(20 / 33)$ & $34 \%(27 / 80)$ \\
\hline Hernigou P 2006 [21] & natural history & 121 & 14 & $77 \%(93 / 121)$ & $75 \%(91 / 121)$ \\
\hline Nam KW 2008 [22] & & 105 & 8.6 & $59 \%(62 / 105)$ & $44 \%(46 / 105)$ \\
\hline Koo KH 1995 [20] & & 19 & 2 & $79 \%(15 / 19)$ & $68 \%(13 / 19)$ \\
\hline Learmonth ID 1990 [23] & $C D^{a}$ & 41 & 2.6 & $83 \%(34 / 41)$ & - \\
\hline Gangji V 2011 [7] & & 11 & 5 & $73 \%(8 / 11)$ & $27 \%(3 / 11)$ \\
\hline Koo KH 1995 [20] & & 18 & 2 & $72 \%(13 / 18)$ & $72 \%(13 / 18)$ \\
\hline Hernigou P 2009 [6] & $C D^{a}+B M C^{b}$ & 534 & 13 & $30 \%(160 / 534)$ & $18 \%(96 / 534)$ \\
\hline Hernigou P 2002 [5] & & 189 & 7 & - & $18 \%(34 / 189)$ \\
\hline Gangji V 2011 [7] & & 13 & 5 & $23 \%(3 / 13)$ & - \\
\hline Kuroda Y 2016 [18] & gelatin hydrogel + FGF & 10 & 1 & $10 \%(1 / 10)$ & $0 \%(0 / 10)$ \\
\hline Lieberman JR 2004 [24] & $C D^{b}+$ fibula allograft $+B M P^{d}$ & 17 & 4.4 & $18 \%(3 / 17)$ & $18 \%(3 / 17)$ \\
\hline Mont A 1996 [25] & osteotomy & 77 & 11.5 & - & $24 \%$ \\
\hline Sugioka Y 1978 [26] & & 41 & 2.5 & $6 \%$ (stage 1,2 ) & - \\
\hline
\end{tabular}

(ONFH-osteonecrosis of the femoral head, ${ }^{a} C D$ Core decompression, ${ }^{\mathrm{b}} B M C$ Bone marrow concentrate, ${ }^{\mathrm{C}} \mathrm{FGF}$ Fibroblast growth factor, ${ }^{\mathrm{d} B M P}$ Bone morphogenetic protein)

somewhat lower than that reported by Hernigou et al. [6]. Hernigou et al. had used a cell separator for the separation of MSCs, while in our study, we used an ordinary blood bag to separate the buffy coat after simple centrifugation. Using this method, we achieved a mean CFU-F count of $2.33 \pm 2.67$ per $10^{6}$ nucleated cells, while Hernigou et al. reported a much higher mean CFU-F count of $12.4 \pm 3.4$ per $10^{6}$ nucleated cells [5]. Despite the difference in CFU-F count, in the pre-collapsed stages, the THA conversion rate did not significantly differ between our study and Hernigou et al's.

Several studies on regenerative therapies (other than bone marrow transplantation) for ONFH have reported lower THA-conversion rates than that observed in our present study [18, 24]. There are also several published studies on bone marrow transplantation. Though positive outcomes of bone marrow transplantation have been reported in systematic reviews, there are no randomized control trials providing level 1 evidence for this therapy [27, 28].

Although, our study showed a better outcome with CABMAT than that observed with the natural progression of ONFH, the overall result is not satisfactory. Cultivation of MSCs, combined transplantation with platelet-rich plasma, filling the core decompression tract with artificial bone for mechanical support, and using a gelated buffy coat in combination with FGF are methods from earlier studies that can be adopted to improve the outcomes of CABMAT $[6,18]$.

Mont et al., Sugioka et al. and Dean et al. reported THA-conversion rates of 24,6 , and $67 \%$ over follow-up periods of $11.5,2.5$, and 8.2 years, respectively, for ONFH treated with femoral osteotomy $[25,29,30]$. The THAconversion rate reported by Sugioka et al. was lower, while the rate reported by Dean et al. was higher than that of our study [30]. However, since the follow-up period and patient backgrounds were different, it is difficult to simply compare the THA conversion rate. Sugioka et al. have suggested that $>36 \%$ of the weight-bearing area should be intact to perform an adequate osteotomy [29]. Therefore, ONFH with a broad necrotic area may not benefit from a femoral osteotomy. Furthermore, osteotomy is a highly technically demanding procedure that can result in an unevenness of the femoral weight-bearing surface. In contrast, our treatment approach does not require technical expertise, and it is cost-effective and minimally invasive.

In the comparative univariate statistical analysis between the THA-conversion and THA non-conversion groups, BMI along with rate of occurrence of stage 4 were found to be significantly higher in the THA-conversion group. In the multivariable statistical analysis, both preoperative stage of ONFH and BMI correlated significantly with conversion to THA. Based on these findings, we suggest that an advanced class and stage of ONFH and a high body weight are predominant risk factors for conversion to THA. Mechanical stress due to body weight is considered as one of the factors responsible for femoral head collapse and conversion to THA. According to a report of Kanazawa et al., in Japan, $\mathrm{BMI} \geq 25$ is defined as obesity [31]. Since the average BMI in the THA-conversion group in this study was 24 $\mathrm{kg} / \mathrm{m}^{2}$, which is lower than the cut-off value for obesity, the extent of the weight's contribution to mechanical stress to the femoral head is unknown. It is known that obesity is closely associated with metabolic syndromes such as diabetes mellitus, hypertension, and hyperlipidemia. But presence rate of metabolic syndromes was not significant different between THA conversion group and THA non-conversion group. While the preoperative 
stage of ONFH is not changeable, body weight is a relatively controllable factor. Regulation of body weight might prevent or delay femoral head collapse and conversion to THA.

There are several limitations to this study. Since this is a retrospective study, having a control group was not possible, which was an important limitation. Secondly, since the etiology, preoperative stages, classification systems, and follow-up periods differed among the study patients, it was difficult to compare the overall effect of CABMAT with the natural progression of ONFH, core decompression, or other treatments.

\section{Conclusion}

Both collapse and THA-conversion rates in the pre-collapse stage of ONFH were estimated at $49 \%(21 / 43)$ and $14 \%$ (6/43), respectively. The overall THA-conversion rate of ONFH was $34 \%(27 / 80)$. Based on our findings, the minimally-invasive and feasible CABMAT therapy can be considered as one of the alternatives for joint-preserving treatment of ONFH, especially in stages 1 and 2.

Our group started CABMAT in 2003. By reporting further long-term results of this therapeutic modality and comparing its outcomes with those of other existing treatments, we aim to ultimately determine the best joint-conserving treatment for ONFH.

\section{Abbreviations}

ARCO: Association Research Circulation Osseous; BMI: Body mass index; CABMAT: Concentrated autologous bone marrow aspirate transplantation; CFU-F: Colony-forming-unit-fibroblast; FGF: Fibroblast growth factor; MRI: Magnetic resonance imaging; MSC: Mesenchymal stem cell; ONFH: Osteonecrosis of the femoral head; THA: Total hip arthroplasty

\section{Acknowledgements}

Not applicable.

\section{Authors' contributions}

YT performed data analysis and participated in the evaluation of femoral head collapse. TY collected data, performed surgeries, contributed to the conception and design of the study, and participated in the evaluation of femoral head collapse. HS, HK, and MY contributed to the conception and design of the study and performed the procedure for concentration of the bone marrow aspirate. $\mathrm{KH}, \mathrm{KA}, \mathrm{HW}, \mathrm{HA}$, and $\mathrm{HM}$ designed the bone marrow transplantation technique and were involved in data analyses. MY and HM supervised the research project. HM also performed the procedures and conducted postoperative evaluations. All authors have read and approved the final manuscript.

\section{Funding}

Not applicable.

\section{Availability of data and materials}

The datasets used and/or analyzed during the current study are available from the corresponding author on reasonable request.

\section{Ethics approval and consent to participate}

Ethical approval for the study design was obtained from the Institutional Ethics Review Committee of the University of Tsukuba. Written informed consent was obtained from all individual participants included in the study.

\section{Consent for publication}

Written informed consent for publication was obtained from the patients included in this study.

\section{Competing interests}

The authors declare that they have no competing interests.

\section{Author details}

${ }^{1}$ Department of Orthopaedic Surgery, Faculty of Medicine, University of Tsukuba, 1-1-1 Tennodai, Tsukuba, Ibaraki 305-8575, Japan. ${ }^{2}$ Division of Regenerative Medicine for Musculoskeletal System, Department of Orthopaedic Surgery, Faculty of Medicine, University of Tsukuba, 1-1-1 Tennodai, Tsukuba, Ibaraki 305-8575, Japan.

Received: 17 May 2019 Accepted: 28 August 2019

Published online: 05 September 2019

\section{References}

1. Takahashi S, Fukushima W, Yamamoto T, Iwamoto Y, Kubo T, Sugano N, et al. Temporal trends in characteristics of newly diagnosed nontraumatic osteonecrosis of the femoral head from 1997 to 2011: a hospital-based sentinel monitoring system in Japan. J Epidemiol. 2015;25:437-44. https://doi.org/10.2188/jea.JE20140162.

2. Yamaguchi R, Yamamoto T, Motomura G, Ikemura S, I Iwamoto Y. Incidence of nontraumatic osteonecrosis of the femoral head in the Japanese population. Arthritis Rheum. 2011;63:3169-73. https://doi.org/10.1002/art.30484.

3. Ikeuchi K, Hasegawa Y, Seki T, Takegami Y, Amano T, Ishiguro N. Epidemiology of nontraumatic osteonecrosis of the femoral head in Japan. Mod Rheumatol. 2015;25:278-81.

4. Saito S, Saito M, Nishina T, Ohzono K, Ono K. Long-term results of total hip arthroplasty for osteonecrosis of the femoral head. A comparison with osteoarthritis. Clin Orthop Relat Res. 1989:198-207 http://www.ncbi.nlm.nih. gov/pubmed/2743660. Accessed 5 May 2019.

5. Hernigou PM, Beaujean FM. Treatment of osteonecrosis with autologous bone marrow grafting. Clincal Orthop Relat Reserch. 2002;405:4-23.

6. Hernigou PM. Cell therapy of hip osteonecrosis with autologous bone marrow grafting. Indian J Orthop. 2009;43:40-5.

7. Gangji V, De Maertelaer V, Hauzeur J-P. Autologous bone marrow cell implantation in the treatment of non-traumatic osteonecrosis of the femoral head: five year follow-up of a prospective controlled study. Bone. 2011:49:1005-9. https://doi.org/10.1016/j.bone.2011.07.032.

8. Sakai S, Mishima H, Ishii T, Akaogi H, Yoshioka T, Uemura T, et al. Concentration of bone marrow aspirate for osteogenic repair using simple centrifugal methods. Acta Orthop. 2008;79:445-8.

9. Sugaya H, Yoshioka T, Kato T, Taniguchi Y, Kumagai H, Hyodo K, et al. Comparative analysis of cellular and growth factor composition in bone marrow aspirate concentrate and platelet-rich plasma. Bone Marrow Res. 2018;2018:1549826. https://doi.org/10.1155/2018/1549826.

10. Hyodo K, Yoshioka T, Sugaya H, Akaogi H, Aoto K, Wada H, et al. Predicting risk factors of total hip arthroplasty conversion after concentrated autologous bone marrow aspirate transplantation for the treatment of idiopathic osteonecrosis of the femoral head: a retrospective review of 213 hips at a mean follow-up of 5 years. J Hip Surg. 2017;01:007-13.

11. Tomaru Y, Yoshioka T, Sugaya H, Shimizu Y, Aoto K, Wada H, et al. Mid-term results of concentrated autologous bone marrow aspirate transplantation for corticosteroid-associated osteonecrosis of the femoral head in systemic lupus erythematosus. Int Orthop. 2018;42:1623-30. https://doi.org/10.1007/ s00264-018-3959-y.

12. Gardeniers JW. A new international classification of osteonecrosis of the ARCO-committee on terminology and classification. ARCO News. 1992;4:416 https://ci.nii.ac.jp/naid/10025588575/. Accessed 28 Sep 2018.

13. Hernigou P, Guerin G, Homma Y, Dubory A, Chevallier N, Rouard H, et al. History of concentrated or expanded mesenchymal stem cells for hip osteonecrosis: is there a target number for osteonecrosis repair? Int Orthop. 2018;42(7):1739-45. https://doi.org/10.1007/s00264-018-4000-1.

14. Aaron RK, Lennox D, Bunce GE. Conservative treatment of osteonecrosis of the femoral head. Comparison of core decompression and pulsing electromagnetic fields. Clin Orthop. 1989;249:209-18.

15. Mankin HJ, Mankin HJ. Nontraumatic necrosis of bone (osteonecrosis). N Engl J Med. 1992;326:1473-9.

16. Cui Q, Wang GJ, Balian G. Steroid-induced adipogenesis in a pluripotential cell line from bone marrow. J Bone Joint Surg Am. 1997;79:1054-63.

17. Hernigou P, Beaujean F, Lambotte JC. Decrease in the mesenchymal stemcell pool in the proximal femur in corticosteroid-induced osteonecrosis. J 
Bone Joint Surg Br. 1999;81:349-55 http://www.ncbi.n/m.nih.gov/pubmed/1 0204950. Accessed 21 Dec 2018.

18. Kuroda Y, Asada R, So K, Yonezawa A, Nankaku M, Mukai K, et al. A pilot study of regenerative therapy using controlled release of recombinant human fibroblast growth factor for patients with pre-collapse osteonecrosis of the femoral head. Int Orthop. 2016;40:1747-54. https://doi.org/10.1007/s00264-015-3083-1.

19. Lee MS, Hsieh PH, Chang YH, Chan YS, Agrawal SUS. Elevated intraosseous pressure in the inter-trochanteric region is associated with poorer results in osteonecrosis of the femoral head treated by multiple drilling. J Bone Jt Surg. 2008;90 ((7):852.

20. Koo KH, Kim R, Ko GH, Song HR, Jeong ST, Cho SH. Preventing collapse in early osteonecrosis of the femoral head. A randomised clinical trial of core decompression. J Bone Joint Surg Br. 1995;77:870-4 http://www.ncbi.nlm. nih.gov/pubmed/7593097. Accessed 15 Nov 2018.

21. Hernigou P, Habibi A, Bachir D, Galacteros F. The natural history of asymptomatic osteonecrosis of the femoral head in adults with sickle cell disease. J Bone Jt Surg. 2006;88:2565-72. https://doi.org/10.2106/JBJS.E.01455.

22. Nam KW, Kim YL, Yoo J, Koo K, Yoon K, Kim H. Fate of untreated asymptomatic osteonecrosis of the femoral head. J Bone Jt Surg Am. 2008;90:477-84.

23. Learmonth ID, Maloon S, Dall G. Core decompression for early atraumatic osteonecrosis of the femoral head. J Bone Joint Surg Br. 1990;72(3):387-90.

24. Lieberman JR, Conduah A, Urist MR. Treatment of osteonecrosis of the femoral head with core decompression and human bone morphogenetic protein. Clin Orthop Relat Res. 2004:139-45 http://www.ncbi.nlm.nih.gov/ pubmed/15577478. Accessed 30 Oct 2018.

25. Mont MA, Fairbank AC, Krackow KA, Hungerford DS. Corrective osteotomy for osteonecrosis of the femoral head: the results of a long-term follow-up study. J Bone Jt Surg. 1996;78:1032-8 http://journals.Iww.com/jbjsjournal/Citation/1 996/07000/Corrective_Osteotomy_for_Osteonecrosis_of_the.7.aspx.

26. Sugioka Y. Transtrochanteric Anterior Rotational Osteotomy of the Femoral Head in the Treatment of Osteonecrosis Affecting the Hip. Clin Orthop Relat Res. 1978:(130):191-201.

27. Papakostidis C, Tosounidis TH, Jones E, Giannoudis PV. The role of "cell therapy" in osteonecrosis of the femoral head. Acta Orthop. 2016;87:72-8. https://doi.org/10.3109/17453674.2015.1077418.

28. Andriolo L, Merli G, Tobar C, Altamura SA, Kon E, Filardo G. Regenerative therapies increase survivorship of avascular necrosis of the femoral head: a systematic review and meta-analysis. Int Orthop. 2018;42:1689-704. https:// doi.org/10.1007/s00264-018-3787-0.

29. Sugioka Y, Hotokebuchi T, Tsutsui H. Transtrochanteric anterior rotationa osteotomy for idiopathic and steroid-induced necrosis of the femoral head: indications and long-term results. Clin Orthop Relat Res. 1992;277:111-20.

30. Dean MT, Cabanela ME. Transtrochanteric anterior rotational osteotomy for avascular necrosis of the femoral head. Long-term results. J Bone Joint Surg Br. 1993;75(4):597-601.

31. Kanazawa M, Yoshiike N, Osaka T, Numba Y Zimmet P, Inoue S. Criteria and classification of obesity in Japan and Asia-Oceania. Asia Pac J Clin Nutr. 2002:11:S732-7. https://doi.org/10.1046/.1440-6047.11.s8.19.x.

\section{Publisher's Note}

Springer Nature remains neutral with regard to jurisdictional claims in published maps and institutional affiliations.

Ready to submit your research? Choose BMC and benefit from:
- fast, convenient online submission
- thorough peer review by experienced researchers in your field
- rapid publication on acceptance
- support for research data, including large and complex data types
- gold Open Access which fosters wider collaboration and increased citations
- maximum visibility for your research: over 100M website views per year
At BMC, research is always in progress.
Learn more biomedcentral.com/submissions

